\title{
Cortical activation during imitative behavior: An fMRI study
}

\author{
Pierpaoli C ${ }^{1}$, Fabri $\mathrm{M}^{1 *}$ and Polonara $\mathrm{G}^{2}$ \\ ${ }^{1}$ Dipartimento di Medicina Sperimentale e Clinica, Università Politecnica delle Marche, Ancona, Italy \\ ${ }^{2}$ Dipartimento di Scienze Cliniche specialistiche e Odontostomatologiche, Università Politecnica delle Marche, Ancona, Italy
}

\begin{abstract}
A previous behavioural study on healthy subjects and callosotomized patients showed that subjects imitated mainly in mirror mode when free to choose the imitation mode, using the right limb to copy a left limb gesture of the facing model; when asked to use the same limb as the model, patients still perform in mirror mode, but controls imitated in anatomical mode, using the right limb for imitating a right limb gesture. These data suggest that the anatomical mode of imitation recruites both hemispheres, being linked to the integrity of the corpus callosum.

The present study aims at investigating with fMRI the neural correlates of imitative perspective-taking, specially the anatomical mode.

Functional MRI was performed in 10 control subjects of the previously tested groups, asked to Observe or Imagine-to-Imitate-with-the-same-limb, in separete runs, intransitive gestures. Different cortical activation in the two conditions were observed: opercular part of left inferior frontal gyrus, left inferior parietal lobule, right temporo-parietal junction and bilateral parietal opercular cortices were activated in Imagine-to-Imitate condition only.
\end{abstract}

Present data confirm previous behavioural observations and indicate that neural circuitry underpinning the anatomical imitation of intransitive gesture likely requires the cooperation of both hemispheres, and therefore the integrity of the corpus callosum.

\section{Introduction}

Imitation is a behavioural event reproducing observed actions. The debate on the function of imitative beahviour, largely diffuse in humans in a variety of tasks and domains, is still animated [1].

Generally, when asked to imitate someone gesturing, an imitator can choose between two modes of imitation acting: a mirror-mode (specular), i.e., using a right limb to copy a spatially matched left limb gesture of the facing model, or an anatomical-mode, i.e. using a right limb for imitating an anatomically matched right limb gesture by the model. In everyday life the actions to be imitated are in the vast majority of cases presented in a third $\left(3^{\text {rd }}\right)$ person persepctive, which is the typical position when the imitator is facing the model [2]. Therefore, in experimental studies aimed at analyzing the behaviour strategies to imitate an action presented in $3^{\text {rd }}$ person, that condition is reproduced.

Data from a previous behavioral study [3] demostrated that healthy subjects tend to prefer the mirror mode imitation when let free to imitate intransitive gestures executed by a model in $3^{\text {rd }}$ personperspective, and to imitate in anatomical mode when asked to perform with the same (or the opposite) limb respect to the model's. Results led to hypothesize that the mirror mode of imitation would recruit the mirror neuron system, whereas the anatomical mode of imitation might be the expression of a different and at least partially independent neural system. In addition, it can be hypothesized that the neural circuits underpinning the anatomical imitation follow a different developing time course, being the imitation ability very early appearing in children [4], but the anatomical perspective very rare [5].

Many papers have been published describing cortical activation observed during the imitative behavior, in healthy adults and children, in patients with brain lesions, psychiatric alterations and also in callosotomized patients (see [6], for a revision of papers on the activation evoked by any kind of gesture; [7], for a revision of the literature describing the activation evoked by imitation of intransitive gestures). The conclusion is that imitation is sustained by a network of brain areas in both hemispheres. In these studies, however, many different protocols have been used, and none of them is comparable with that used in our previous behavioural analysis. We decided therefore to design a new study by presenting to a group of healthy subjects, selected from the group who participated in previous behavioural study [3], a stimulation protocol based on that used in our previous behavioral studies $[3,8]$, to make possible a direct comparison between behavioral and functional observations, obtained from the same people.

The main goal of the present research was to study with fMRI the cortical activation pattern evoked in the two conditions, i.e., mirror and anatomical imitation of intransitive gestures, to compare the behavioural data obtained in our previous studies [3] with the functional data collected in the present work. Since in the magnet it is necessary to reduce as much as possible any movement, and the subjects could not be asked to actually imitate the model's gestures, some adaptation

*Correspondence to: Mara Fabri, Dipartimento di Medicina Sperimentale e Clinica, Sezione di Neuroscienze e Biologia Cellulare, Facoltà di Medicina, Università Politecnica delle Marche, Via Tronto 10/A, 60020 Ancona, Italy, E-mail: m.fabri@univpm.it

Key words: anatomical perspective, corpus callosum, imitation, intransitive gestures, mirror-mode perspective

Received: March 25, 2020; Accepted: April 13, 2020; Published: April 23, 2020 
was made to the experimental protocol: the free imitation session was substitute by an observation session, by assuming that simple gesture observation does activate the cortical pattern for the mirror imitation; the driven imitation session was replaced by an imaging of imitation with the same limb, since healthy subjects interpreted the instruction "same limb" according to an anatomical criterion [3], and performed the imitation with anatomical mode in the behavioural setting. At variance with previous researches, our subjects were instructed on the way they had to imagine to imitate, i.e., "use the same limb of the model". In a previous paper, it has been shown that this instruction induces healthy subjects to imitate intransitive gestures with an anatomical perspective [3]. Therefore, it can be expected that healthy subjects, when asked to image to imitate "using the same limb as the model", image to perform in anatomical perspective.

The present study was carried out with functional magnetic resonance imaging (fMRI), and was aimed to identify, in healthy control subjects:

1. the cortical areas activated during the observation of intransitive meaningful gestures performed by a model, presented in video clips in $3^{\text {rd }}$ person perspective (condition likely simulating the mirror mode imitation);

2. the cortical areas activated by imaging to imitate intransitive meaningful gestures performed by a $3^{\text {rd }}$ person model, using the same limb of the model (condition likely simulating the anatomical mode imitation).

The choice of presenting meaningful gestures is because in our previous behavioural study no differences in performance were found between meaningful versus meaningless gestures, in terms of imitative perspective [3]. Then, since meaningful gestures are known actions, it is probably easier to imagine to imitate them. The instruction "imitate with the same limb as the model" was selected in that in previous studies this specific instruction gave different results between healthy control subjects and callosotomized patients [8], suggesting that interpretation of the word "same" was different in the two groups of individuals, and that the meaning could be dependent on the integrity of interhemispheric connections.

The results were that the sole observation of intransitive gestures activated the cortical circuitry of the mirror neuron system (MNS; [911 , and the cortical areas involved in the planning the execution of the voluntary movements [12]. The imagery imitation of the gestures with the same limb as the model (IIMsL) evoked a broader and greater activation pattern [13], involving cortical areas of both hemispheres; namely, during this second task, areas belonging to the MNS were recruited, together with areas probably afferent to the Theory of Mind (such as TPJ; [14,15], and areas involved in the mental rotation mechanism $[16,17]$. Some of the results have been presented as short communications [18-20].

\section{Experimental procedures}

Participants: The data were collected from 10 healthy volunteers (right handed; 5 males; mean age 32 years, $\mathrm{SD}=6,7$; Table 1 ). Handedness was evaluated by the Oldfield inventory [21]. All subjects gave their informed consent to participate in the study. The experimental protocol was approved by the Ethics Committee of Università Politecnica delle Marche (Ancona, Italy).

Stimuli and tasks: The stimuli were $12 \mathrm{~s}$ video clips showing a model in $3^{\text {rd }}$ person perspective performing two intransitive gestures
Table 1. Characteristics of the subjects participating in the study

\begin{tabular}{|c|c|c|c|}
\hline & Age & Gender & $\begin{array}{c}\text { Handedness } \\
\text { (Oldfield score) }\end{array}$ \\
\hline C1 & 33 & M & Right (11/50) \\
\hline C2 & 37 & M & Right $(12 / 50)$ \\
\hline C3 & 48 & M & Right $(13 / 50)$ \\
\hline C4 & 25 & M & Right $(18 / 50)$ \\
\hline C5 & 33 & M & Right $(13 / 50)$ \\
\hline C6 & 30 & F & Right $(11 / 50)$ \\
\hline C7 & 28 & F & Right $(14 / 50)$ \\
\hline C8 & 28 & F & Right $(14 / 50)$ \\
\hline C9 & 27 & F & Right $(14 / 50)$ \\
\hline C10 & 30 & F & Right $(13 / 50)$ \\
\hline
\end{tabular}

(Figure 1). They were edited using E-PRIME software (Psychology Software Tools Inc., Pittsburgh, PA), and were presented during the fMRI session according to a block-designed protocol alternating 12 -s-periods of rest and stimulation.

The video clips were selected among the stimuli series used in the previous behavioural study [3], showing a model executing intransitive gestures with her upper limbs: one of the two gestures was a bodyrelated gesture (silence gesture: closed hand with index finger upright, close to the lips; Figures $1 \mathrm{~A}$ and $1 \mathrm{C}$ ), and the other was a body-unrelated gesture (bye-bye gesture: open hand, waving $45^{\circ}$ to one side and the other, repeatedly; Figures 1B and 1D). Each gesture was performed with either right or left limb in separate stimulation periods (blocks).

Each 5-min functional run started with a $12 \mathrm{~s}$ rest period (baseline), in which a fixation cross was presented in the center of a grey background, followed by the first task period. The video clips showing the four gestures (silence, model's right hand; bye-bye, model's left hand; silence, model's left hand; bye-bye, model's right hand) were presented each in a 12-s task period, alternated to a 12-s rest period; during each 12-s task period, the same video clip, depicting the same gesture, was flashed 4 times.

At the beginning of the video clip both model's arms were relaxed along the body, then the gesture was executed by the model who then returned to the initial standing position.

The following two conditions were investigated:

1. Observation (OBS): the subject was required to carefully observe the video clips. The instruction, in italian, was: "Please, simply look at the video".

2. Imitative imagery (IIMsL): the subject observed the videos and was requested to imagine himself performing the movement with the same limb used by the model. The instruction, in italian, was "Please, as soon as you see the gesture of the model, imagine to reproduce the same gesture with the same limb used by the model".

Functional MRI stimulation protocol: Before the scanning sessions, subjects received verbal information about the experiment. The fMRI sessions consisted of 2 functional blocks, each containing 13 resting periods alternating with 12 stimulation periods. Each functional block started and ended with a resting period; during each stimulation period a single gesture was flashed 4 times, lasting each $3 \mathrm{sec}$ (Figure 1). The stimulation blocks were presented within the run in the following sequence: Silence Right (SR; Figure 1A), Bye-bye Left (BL; Figure 1B), Silence Left (SL; Figure 1C) and Bye-bye Right (BR; Figure 1D). 


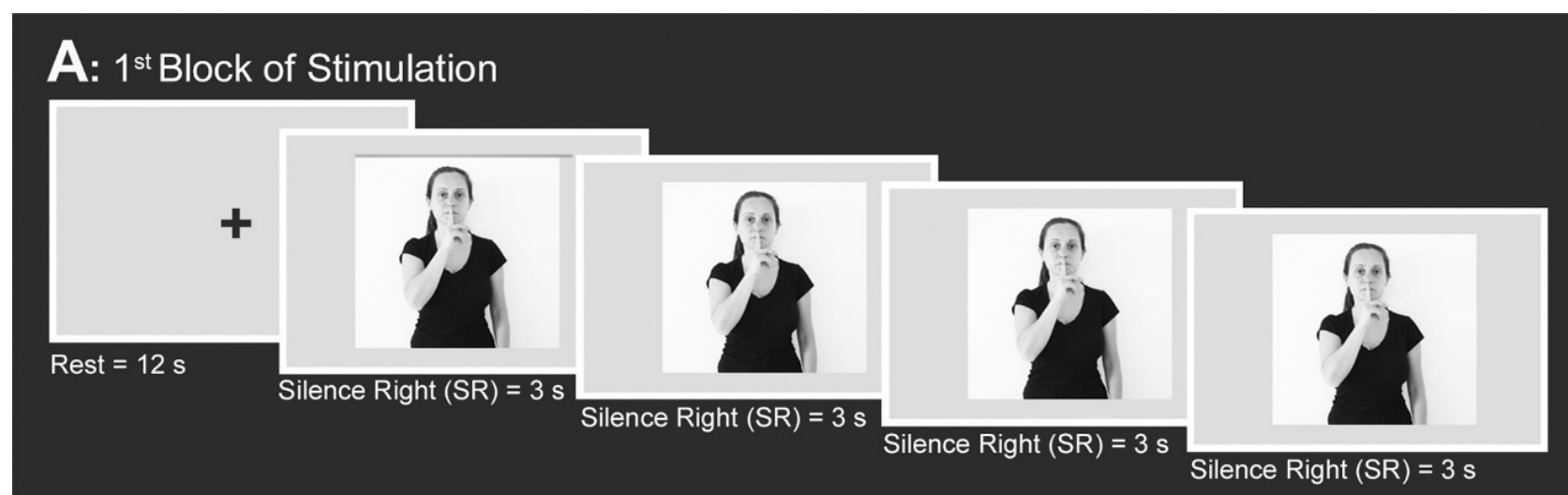

\section{B: $2^{\text {nd }}$ Block of Stimulation}

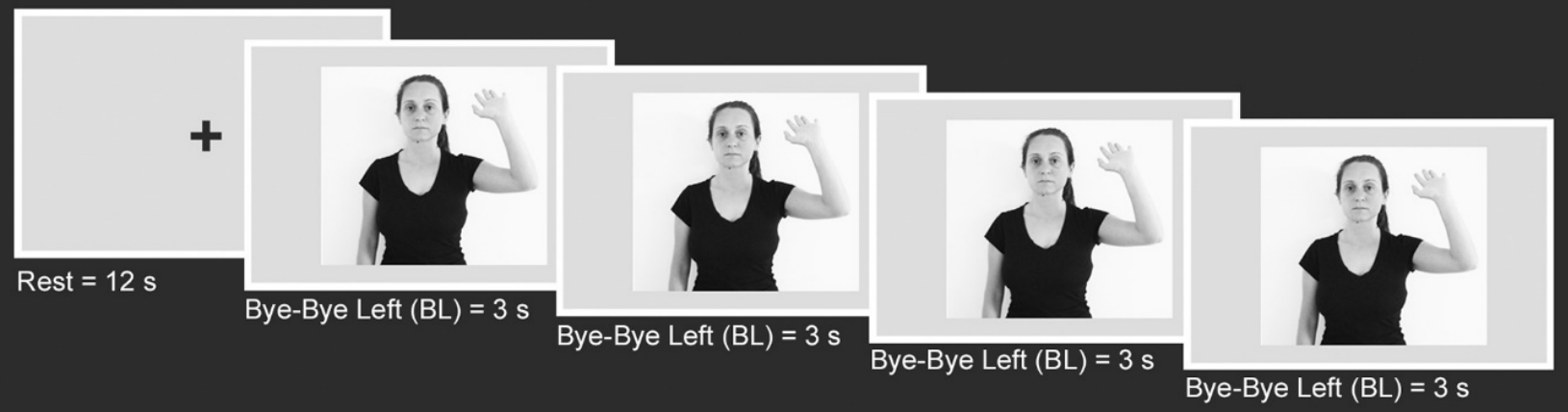

C: $3^{\text {rd }}$ Block of Stimulation

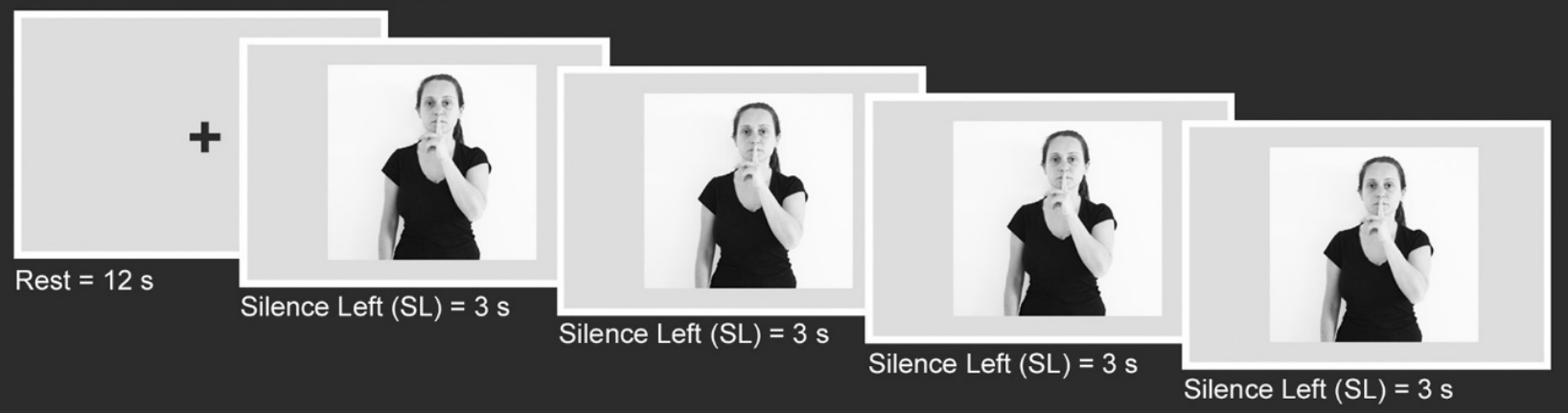

\section{D: $4^{\text {th }}$ Block of Stimulation}

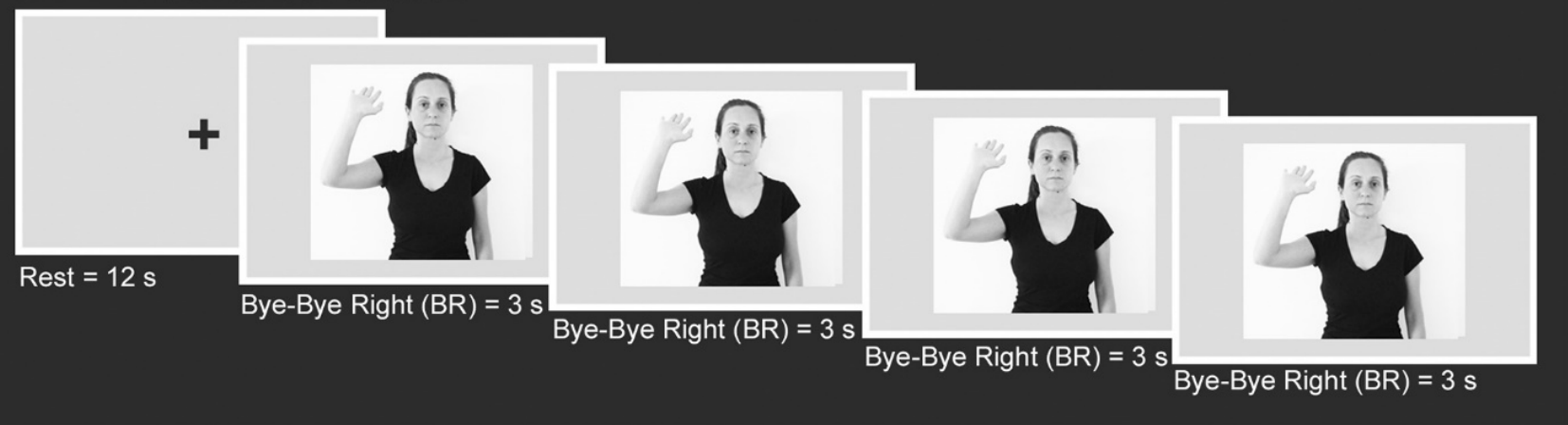

Figure 1. Functional MRI design. The functional design consisted of 2 identical runs composed of 13 resting periods alternating with 12 stimulation periods (blocks). The stimulation blocks were presented in the following order: 1. silence gesture executed by the model with her right limb (SR; A); 2. bye-bye gesture executed by the model with her left limb (BL; B); 3. silence gesture executed by the model with her left limb (SL; C); 4 . bye-bye gesture executed by the model with her right limb (BR; D). During each stimulation bloch each image was flashed 4 times for $3 \mathrm{~s}$. Blocks were not randomzied 
At the end of the whole scanning session, the subjects were asked about the limb (right or left) they imagined to use to imitate the model's gesture: all of them answered to have imagined to imitate by using their right limb when the model used her right limb, and vice versa, indicating they adopted an anatomical imitation strategy.

\section{Functional MRI data acquisition}

Data were collected using a $1.5 \mathrm{~T}$ (Signa Excite NV/i CV/i, General Electric Medical System, Milwaukee, WI, USA) equipped with $50 \mathrm{mT} / \mathrm{m}$ gradients. Subjects, with their head restrained within a circularly polarized head coil, were invited to wear $3 \mathrm{D}$ glasses, to lie down in a supine position, avoiding even minimal movement. The videos were presented through VisualStim Digital glasses (Resonance Technology, Inc.) that each participant wore before entering the scanner. To reduce head motion artifacts during the data acquisition, a custom-head support was used.

\section{Image acquisition occurred through 4 steps:}

1. acquisition of anatomical three-plane localizer (2D SPGR, TR $120 \mathrm{~ms}$, TE $15 \mathrm{~ms}$, Flip Angle $70^{\circ}$, FOV $23 \times 23 \mathrm{~cm}$, slice thickness $5 \mathrm{~mm}$, Matrix $256 \times 256,1 \mathrm{Nex}$, scan time $31 \mathrm{~s}$ );

2. acquisition of a $3 \mathrm{D}$ data set (IR Prep Fast SPGR; TR $15.2 \mathrm{~ms}$, TE $6.9 \mathrm{~ms}$, TI $500 \mathrm{~ms}$, Flip Angle $15^{\circ}$, FOV $29 \times 29 \mathrm{~cm}$, slice thickness $1 \mathrm{~mm}$, Matrix $288 \times 288,1 \mathrm{Nex}$, scan time $8: 20 \mathrm{~min}$ );

3. acquisition of 20 contiguous 5 -mm-thick axial or oblique functional images with a single-shot $\mathrm{T} 2{ }^{\star}$-weighted gradient-echo EPI sequence (TR $3000 \mathrm{~ms}$, TE $60 \mathrm{~ms}$, Flip Angle $90^{\circ}$, FOV $28 \times 21 \mathrm{~cm}$, Matrix $96 \times 64,1 \mathrm{Nex}$, scan time 5:12 min);

4. high-resolution axial (or oblique) anatomical images acquired from 20 selected planes (2D SPGR, TR $100 \mathrm{~ms}$, TE $12 \mathrm{~ms}$, Flip Angle $70^{\circ}$, FOV $28 \times 21 \mathrm{~cm}$, thickness $5 \mathrm{~mm}$, Matrix $256 \times 256,1$ Nex, scan time 2:25 min for 20 images) to superimpose functional activation images onto the anatomical landmarks, allowing to show blood vessels considered as possible sources of BOLD signals.

Two thousand axial or oblique functional images (100 per section, 1 image/3 s) were acquired during the stimulation cycle from 20 contiguous 5 -mm-thick axial sections obtained from 20 previously selected planes. Functional images were obtained with the BOLD method. The axial planes were orthogonal to both the sagittal and the coronal planes, and their orientation was parallel to the AC-PC line.

Images were then transferred to a Unix workstation (General Electric Advantage Windows 4.2) and finally to a computer to be analyzed.

\section{Functional MRI data analysis}

BrainVoyager software package (BrainVoyagerQX, Version 2.3.1.1770, 32-bit, Copyright ( $2001-2014$ Rainer Goebel) was used for analyzing data (DICOM format) that were loaded and converted into BrainVoyager's internal ".fmr" data format.

Intensity inhomogeneity correction (IIHC) BrainVoyager tool was applied and a standard sequence of preprocessing steps performed. As it is impossible to lie completely still during a time interval the entire scanning session, and since physiological as well as physical (scannerrelated) noise can reduce substantially the power of statistical data analysis, in order to minimize the false positive activations while increasing sensitivity to true task-related activations, slice scan timing (sinc interpolation based on information about the TR $=3000 \mathrm{~ms}$ ), 3D correction for motion artifacts and temporal filtering were applied. No low-pass temporal or spatial filters were used, as the False Discovery Rate approach used for the specification of an appropriate threshold of statistical maps avoided spatial smoothing. After creating a functional project from measured DICOM files, the original voxel data were stored in STC (slice time course) files.

For each subject, the functional images relative to each functional run were co-registrated and aligned to the three-dimensional high resolution images and finally transformed into Talairach space [22]. The first two images of each functional series were discarded to take into account signal intensity variations due to progressive saturation.

In order to investigate BOLD signals relative to the two functional runs (OBS and IIMsL) and for the baseline (R) single-subjects contrasts for each run were followed by a second step analysis, consisting a multisubjects analysis.

First, to determine whether gestures observation (OBS) differed from imaginating to mentally reproduce the gestures with the same limb as the model (IIMsL), a General Linear Model (GLM) approach was used to generate statistical parametric maps. In single-subject analysis GLMs, the predictor time courses were convolved with a standard hemodynamic response function (HRF) to account for the hemodynamic delay. After computing statistical maps for each individual, the volume time course (VTC) files from multiple subjects were submitted to multi-subjects analyses where the statistical maps containing estimated effects (beta values) separately for each subject were the inputs.

Activation foci were studied by selecting Regions-Of-Interest (ROIs) in frontal, parietal and temporal cortices. The False Discovery Rate approach (FDR; for multiple comparison), by detecting voxels with a high sensitivity (if there are true effects in the data) was used for the specification of an appropriate threshold of statistical maps. Regions activated were identified as significant at FDR $q=0.001$, with a cluster size equal or exceeding 10 contigous activated voxels. Additional analyses were performed with less selective, but still significant, values of $\mathrm{q}$, which were $0.005,0.01$ and 0.05 . In the subtraction analysis (IIMsL $>$ OBS), regions activated were identified as significant at FDR $\mathrm{q}=0.05$, with a cluster size equal or exceeding 3 contiguous activated voxels. Additional analyses were performed without FDR statistical approach, to evidence also smaller activations; in those cases, the $\mathrm{p}$ values were $\leq 0.01$ or $\leq 0.05$.

Under the assumption that voxels (or vertices) with the same coordinates in different brains access corresponding brain regions, in order to integrate the data from multiple subjects into a single GLM analysis, i.e., to achieve better comparison across voxels and to normalize the variance of the individual runs, raw fMRI time course z-normalization was calculated. When the activation coincided with the stimulation pattern, it was assumed to be evoked by the specific predictor.

Contrasts were used to test for differences between each of the two experimental conditions (OBS and IIMsL) and the baseline, and between the IIMs Land OBS conditions.

\section{Results}

This study aimed at defining the cortical areas activated in OBS and IIMsL runs. Using a subtraction method, BOLD signals intensity differences (contrasts) between each run and baseline (OBS>baseline and IIMsL $>$ baseline) and between the two runs (IIMsL $>$ OBS) were 
observed. The activation threshold was kept very selective to be sure that all activations observed were due to the specific task.

Separated analysis was performed for each of the followng conditions (c):

$\mathrm{cl}=$ All gestures together

c2 $=$ Gestures executed by the model with her right limb

c3 $=$ Gestures executed by the model with her left limb

c4 =Bye-bye gesture executed by the model with her right limb

c5 =Bye-bye gesture executed by the model with her left limb

c6 $=$ Silence gesture executed by the model with her right limb

c7 =Silence gesture executed by the model with her left limb
The description will be provided for all conditions in the text. Data tables are reported in the text only for condition $\mathrm{c} 1$ (=all gestures), c2 (=gestures executed by the model with her right limb), c3 (=gestures executed by the model with her left limb).

\section{Brain areas activated during OBS task}

During OBS run, the contrast task OBS versus baseline resulted in map of active voxel clusters, whose Talairach coordinates [22] are reported in Tables 2 and 3. Multisubject analysis generated 41736 active voxels, to which the FDR statistical approach was applied, with $\mathrm{q}=0.001$ and cluster threshold $=10$ voxels. Additional analyses were performed with less selective, but still significant, values of $\mathrm{q}$, which was $0.005,0.01$ and 0.05 . In Table 3 , the results obtained with less selective q values are evidenced.

Table 2. Cortical areas activated in all subjects in OBS and IIMsL

\begin{tabular}{|c|c|c|c|c|c|c|c|c|c|c|c|c|c|c|c|}
\hline \multirow{3}{*}{\begin{tabular}{|c|} 
All gestures, condition 1 \\
Cortical Area \\
\end{tabular}} & \multirow[b]{3}{*}{ BA } & \multicolumn{4}{|c|}{$\begin{array}{c}\text { OBSERVE } \\
\text { FDR } q=0.001\end{array}$} & \multirow[b]{3}{*}{ BA } & \multicolumn{4}{|c|}{$\begin{array}{l}\text { IMAGE TO IMITATE } \\
\text { FDR } q=0.001\end{array}$} & \multirow[b]{3}{*}{ BA } & \multicolumn{4}{|c|}{$\begin{array}{l}\text { IMAGE }>\text { OBSERVE } \\
\text { FDR } q=0.05\end{array}$} \\
\hline & & \multirow[b]{2}{*}{$\mathbf{H}$} & \multicolumn{3}{|c|}{ Talairach coordinates } & & \multirow[b]{2}{*}{$\mathbf{H}$} & \multicolumn{3}{|c|}{ Talairach coordinates } & & \multirow[b]{2}{*}{$\mathbf{H}$} & \multicolumn{3}{|c|}{ Talairach coordinates } \\
\hline & & & $\mathrm{x}$ & $\mathrm{y}$ & $\mathrm{z}$ & & & $\mathrm{x}$ & $\mathrm{y}$ & $\mathrm{z}$ & & & $\mathrm{x}$ & $\mathrm{y}$ & $\mathrm{z}$ \\
\hline \multicolumn{16}{|l|}{ FRONTAL LOBE } \\
\hline \multirow[t]{2}{*}{ Medial Frontal Gyrus (MFG) } & 6 & $\mathbf{R}$ & & & & 6 & $\mathbf{R}$ & 3 & -5 & 58 & 6 & $\mathbf{R}$ & & & \\
\hline & & $\mathbf{L}$ & -4 & 4 & 49 & & $\mathbf{L}$ & -2 & -9 & 58 & & $\mathbf{L}$ & -3 & -18 & 60 \\
\hline \multirow[t]{2}{*}{ Inferior Frontal Gyrus (IFG) } & 9 & $\mathbf{R}$ & 44 & 3 & 32 & 9 & $\mathbf{R}$ & 29 & 31 & 27 & 9 & $\mathbf{R}$ & & & \\
\hline & & $\mathbf{L}$ & & & & & $\mathbf{L}$ & -39 & 30 & 27 & & $\mathbf{L}$ & & & \\
\hline \multirow[t]{2}{*}{ Inferior Frontal Gyrus (IFG) } & 44 & $\mathbf{R}$ & & & & 44 & $\mathbf{R}$ & 49 & 8 & 8 & 44 & $\mathbf{R}$ & & & \\
\hline & & $\mathbf{L}$ & & & & & $\mathbf{L}$ & -54 & 4 & 8 & & $\mathbf{L}$ & -52 & 7 & 6 \\
\hline \multirow[t]{2}{*}{ Inferior Frontal Gyrus (IFG) } & 45 & $\mathbf{R}$ & & & & 45 & $\mathbf{R}$ & 44 & 17 & 3 & 45 & $\mathbf{R}$ & & & \\
\hline & & $\mathbf{L}$ & & & & & $\mathbf{L}$ & & & & & $\mathbf{L}$ & & & \\
\hline \multirow[t]{2}{*}{ Inferior Frontal Gyrus (IFG) } & 46 & $\mathbf{R}$ & & & & 46 & $\mathbf{R}$ & & & & 46 & $\mathbf{R}$ & & & \\
\hline & & $\mathbf{L}$ & & & & & $\mathbf{L}$ & -43 & 43 & 9 & & $\mathbf{L}$ & & & \\
\hline Precentral Gyrus (PrG) & 6 & $\mathbf{R}$ & 41 & -5 & 49 & 6 & $\mathbf{R}$ & 43 & -2 & 43 & 6 & $\mathbf{R}$ & & & \\
\hline & & $\mathbf{L}$ & -41 & -6 & 47 & & $\mathbf{L}$ & -42 & -6 & 43 & & $\mathbf{L}$ & & & \\
\hline PARIETAL LOBE & & & & & & & & & & & & & & & \\
\hline Parietal lobe, precuneus & 7 & $\mathbf{R}$ & & & & 7 & $\mathbf{R}$ & & & & 7 & $\mathbf{R}$ & & & \\
\hline & & $\mathbf{L}$ & -18 & -77 & 41 & & $\mathbf{L}$ & -2 & -70 & 51 & & $\mathbf{L}$ & & & \\
\hline Superior parietal lobule (SPL) & 7 & $\mathbf{R}$ & 24 & -57 & 44 & 7 & $\mathbf{R}$ & 32 & -65 & 47 & 7 & $\mathbf{R}$ & & & \\
\hline & & $\mathbf{L}$ & -36 & -51 & 44 & & $\mathbf{L}$ & -30 & -70 & 47 & & $\mathbf{L}$ & & & \\
\hline Inferior Parietal Lobule (IPL) & 40 & $\mathbf{R}$ & & & & 40 & $\mathbf{R}$ & 41 & -46 & 47 & 40 & $\mathbf{R}$ & & & \\
\hline & & $\mathbf{L}$ & -43 & -45 & 44 & & $\mathbf{L}$ & -39 & -56 & 47 & & $\mathbf{L}$ & -47 & -25 & 15 \\
\hline TEMPORAL LOBE & & & & & & & & & & & & & & & \\
\hline Superior temporal gyrus (STG) & 22 & $\mathbf{R}$ & 47 & -42 & 7 & 22 & $\mathbf{R}$ & 45 & -44 & 12 & 22 & $\mathbf{R}$ & 48 & 11 & -3 \\
\hline & & $\mathbf{L}$ & & & & & $\mathbf{L}$ & -56 & -42 & 12 & & $\mathbf{L}$ & & & \\
\hline Temp Lobe, sup temp gyrus & 39 & $\mathbf{R}$ & 47 & -47 & 8 & 39 & $\mathbf{R}$ & 40 & -51 & 7 & 39 & $\mathbf{R}$ & & & \\
\hline & & $\mathbf{L}$ & -50 & -48 & 2 & & $\mathbf{L}$ & -50 & -54 & 7 & & $\mathbf{L}$ & & & \\
\hline INSULAR LOBE & & & & & & & & & & & & & & & \\
\hline Insula (Ins) middle & 13 & $\mathbf{R}$ & & & & 13 & $\mathbf{R}$ & 44 & -1 & 6 & 13 & $\mathbf{R}$ & 44 & -1 & 6 \\
\hline & & $\mathbf{L}$ & & & & & $\mathbf{L}$ & -46 & -2 & 6 & & $\mathbf{L}$ & -44 & -3 & 6 \\
\hline Insula (Ins) posterior & 13 & $\mathbf{R}$ & 44 & -39 & 21 & 13 & $\mathbf{R}$ & 44 & -39 & 21 & 13 & $\mathbf{R}$ & & & \\
\hline & & $\mathbf{L}$ & -49 & -44 & 22 & & $\mathbf{L}$ & -49 & -44 & 22 & & $\mathbf{L}$ & & & \\
\hline OCCPITAL LOBE & & & & & & & & & & & & & & & \\
\hline Cuneus (Cun) & 17 & $\mathbf{R}$ & 12 & -94 & 1 & 17 & $\mathbf{R}$ & 12 & -94 & 1 & 17 & $\mathbf{R}$ & & & \\
\hline & & $\mathbf{L}$ & -12 & -94 & 1 & & $\mathbf{L}$ & -12 & -94 & 1 & & $\mathbf{L}$ & & & \\
\hline Cuneus (Cun) & 18 & $\mathbf{R}$ & 10 & -91 & 18 & 18 & $\mathbf{R}$ & 10 & -91 & 18 & 18 & $\mathbf{R}$ & & & \\
\hline & & $\mathbf{L}$ & -9 & -92 & 18 & & $\mathbf{L}$ & -9 & -92 & 18 & & $\mathbf{L}$ & & & \\
\hline Lingual Gyrus (LgG) & 18 & $\mathbf{R}$ & 24 & -85 & -9 & 18 & $\mathbf{R}$ & 20 & -77 & -9 & 18 & $\mathbf{R}$ & & & \\
\hline & & $\mathbf{L}$ & -25 & -86 & -9 & & $\mathbf{L}$ & -20 & -77 & -9 & & $\mathbf{L}$ & & & \\
\hline Middle Occipital Gyrus (MOG) & 19 & $\mathbf{R}$ & 47 & -61 & 6 & 19 & $\mathbf{R}$ & 47 & -61 & 6 & 19 & $\mathbf{R}$ & & & \\
\hline & & $\mathbf{L}$ & -46 & -69 & 6 & & $\mathbf{L}$ & -46 & -69 & 6 & & $\mathbf{L}$ & & & \\
\hline Middle Occipital Gyrus (MOG) & 37 & $\mathbf{R}$ & 44 & -64 & -3 & 37 & $\mathbf{R}$ & 44 & -64 & -3 & 37 & $\mathbf{R}$ & & & \\
\hline & & $\mathbf{L}$ & -44 & -69 & 0 & & $\mathbf{L}$ & -44 & -69 & 0 & & $\mathbf{L}$ & & & \\
\hline Corpus Callosum (CC) non fdr, $p<0.035$ & & & & & & & & -1 & 0 & 23 & & & 1 & 16 & 19 \\
\hline
\end{tabular}


Table 3. Cortical areas activated in OBS

\begin{tabular}{|c|c|c|c|c|c|c|c|c|c|c|c|c|c|c|c|}
\hline \multirow{3}{*}{\begin{tabular}{|r|} 
OBSERVE, FDR q $=0.001$ \\
\\
Cortical Area \\
\end{tabular}} & \multirow[b]{3}{*}{ BA } & \multicolumn{4}{|c|}{ All gestures condition 1} & \multirow[b]{3}{*}{ BA } & \multicolumn{4}{|c|}{ Model's RL gestures condition 2} & \multicolumn{5}{|c|}{ Model's LL gestures condition 3} \\
\hline & & \multirow[b]{2}{*}{ H } & \multicolumn{3}{|c|}{ Talairach coordinates } & & \multirow[b]{2}{*}{ H } & \multicolumn{3}{|c|}{ Talairach coordinates } & \multirow[b]{2}{*}{ BA } & \multirow[b]{2}{*}{$\mathbf{H}$} & \multicolumn{3}{|c|}{ Talairach coordinates } \\
\hline & & & $\mathrm{x}$ & $\mathrm{y}$ & $\mathrm{z}$ & & & $\mathrm{x}$ & $\mathrm{y}$ & $\mathrm{z}$ & & & $\mathrm{x}$ & $\mathrm{y}$ & $\mathrm{z}$ \\
\hline \multicolumn{16}{|l|}{ FRONTAL LOBE } \\
\hline \multirow{2}{*}{ Medial Frontal Gyrus (MFG) } & 6 & $\mathbf{R}$ & & & & 6 & $\mathbf{R}$ & & & & 6 & $\mathbf{R}$ & & & \\
\hline & & $\mathbf{L}$ & -4 & 4 & 49 & & $\mathbf{L}$ & -4 & 4 & 49 & & $\mathbf{L}$ & -4 & 4 & 49 \\
\hline \multirow[t]{2}{*}{ Inferior Frontal Gyrus (IFG) } & 9 & $\mathbf{R}$ & 44 & 3 & 32 & 9 & $\mathbf{R}$ & 44 & 3 & 32 & 9 & $\mathbf{R}$ & 44 & 3 & 32 \\
\hline & & $\mathbf{L}$ & & & & & $\mathbf{L}$ & & & & & $\mathbf{L}$ & & & \\
\hline \multirow[t]{2}{*}{ Inferior Frontal Gyrus (IFG) } & 45 & $\mathbf{R}$ & & & & 45 & $\mathbf{R}$ & & & & 45 & $\mathbf{R}$ & & & \\
\hline & & $\mathbf{L}$ & & & & & $\mathbf{L}$ & & & & & $\mathbf{L}$ & & & \\
\hline \multirow[t]{2}{*}{ Precentral Gyrus (PrG) } & 6 & $\mathbf{R}$ & 41 & -5 & 49 & 6 & $\mathbf{R}$ & & & & 6 & $\mathbf{R}$ & & & \\
\hline & & $\mathbf{L}$ & -41 & -6 & 47 & & $\mathbf{L}$ & & & & & $\mathbf{L}$ & & & \\
\hline \multicolumn{16}{|l|}{ PARIETAL LOBE } \\
\hline \multirow[t]{2}{*}{ Parietal lobe, precuneus } & 7 & $\mathbf{R}$ & & & & 7 & $\mathbf{R}$ & & & & 7 & $\mathbf{R}$ & & & \\
\hline & & $\mathbf{L}$ & -18 & -77 & 41 & & $\mathbf{L}$ & & & & & $\mathbf{L}$ & -20 & -79 & 39 \\
\hline \multirow[t]{2}{*}{ Superior parietal lobule (SPL) } & 7 & $\mathbf{R}$ & 24 & -57 & 44 & 7 & $\mathbf{R}$ & & & & 7 & $\mathbf{R}$ & & & \\
\hline & & $\mathbf{L}$ & -36 & -51 & 44 & & $\mathbf{L}$ & & & & & $\mathbf{L}$ & & & \\
\hline Inferior Parietal Lobule (IPL) & 40 & $\mathbf{R}$ & & & & 40 & $\mathbf{R}$ & & & & 40 & $\mathbf{R}$ & & & \\
\hline & & $\mathbf{L}$ & -43 & -45 & 44 & & $\mathbf{L}$ & & & & & $\mathbf{L}$ & & & \\
\hline TEMPORAL LOBE & & & & & & & & & & & & & & & \\
\hline Superior temporal gyrus (STG) & 22 & $\mathbf{R}$ & 47 & -42 & 7 & 22 & $\mathbf{R}$ & 45 & -44 & 8 & 22 & $\mathbf{R}$ & 47 & -44 & 11 \\
\hline & & $\mathbf{L}$ & & & & & $\mathbf{L}$ & -50 & -47 & 15 & & $\mathbf{L}$ & 53 & -47 & 15 \\
\hline Superior temporal gyrus (STG) & 39 & $\mathbf{R}$ & 47 & -47 & 8 & 22 & $\mathbf{R}$ & 48 & -42 & -1 & 22 & $\mathbf{R}$ & 47 & -44 & 11 \\
\hline & & $\mathbf{L}$ & -50 & -48 & 2 & & $\mathbf{L}$ & & & & & $\mathbf{L}$ & & & \\
\hline INSUAR LOBE & & & & & & & & & & & & & & & \\
\hline Insula (Ins) middle & 13 & $\mathbf{R}$ & & & & 13 & $\mathbf{R}$ & & & & 13 & $\mathbf{R}$ & & & \\
\hline & & $\mathbf{L}$ & & & & & $\mathbf{L}$ & -40 & 13 & -2 & & $\mathbf{L}$ & & & \\
\hline Insula (Ins) posterior & 13 & $\mathbf{R}$ & 44 & -39 & 21 & 13 & $\mathbf{R}$ & 44 & -40 & 20 & 13 & $\mathbf{R}$ & 45 & -38 & 20 \\
\hline & & $\mathbf{L}$ & -49 & -44 & 22 & & $\mathbf{L}$ & -51 & -48 & 20 & & $\mathbf{L}$ & -48 & -44 & 20 \\
\hline OCCIPITAL LOBE & & & & & & & & & & & & & & & \\
\hline Cuneus (Cun) & 17 & $\mathbf{R}$ & 12 & -94 & 1 & 17 & $\mathbf{R}$ & 12 & -94 & 1 & 17 & $\mathbf{R}$ & 11 & -92 & 1 \\
\hline & & $\mathbf{L}$ & -12 & -94 & 1 & & $\mathbf{L}$ & -12 & -94 & 1 & & $\mathbf{L}$ & -11 & -92 & 1 \\
\hline Cuneus (Cun) & 18 & $\mathbf{R}$ & 10 & -91 & 18 & 18 & $\mathbf{R}$ & 8 & -91 & -1 & 18 & $\mathbf{R}$ & 11 & -91 & 15 \\
\hline & & $\mathbf{L}$ & -9 & -92 & 18 & & $\mathbf{L}$ & -10 & -93 & -1 & & $\mathbf{L}$ & -11 & -91 & 15 \\
\hline Lingual Gyrus (LgG) & 18 & $\mathbf{R}$ & 24 & -85 & -9 & 18 & $\mathbf{R}$ & 24 & -85 & -9 & 18 & $\mathbf{R}$ & 24 & -85 & -9 \\
\hline & & $\mathbf{L}$ & -25 & -86 & -9 & & $\mathbf{L}$ & -25 & -86 & -9 & & $\mathbf{L}$ & -25 & -86 & -9 \\
\hline Middle Occipital Gyrus (MOG) & 19 & $\mathbf{R}$ & 47 & -61 & 6 & 19 & $\mathbf{R}$ & 46 & -60 & -1 & 19 & $\mathbf{R}$ & 46 & -60 & -1 \\
\hline & & $\mathbf{L}$ & -46 & -69 & 6 & & $\mathbf{L}$ & -48 & -65 & -1 & & $\mathbf{L}$ & -48 & -65 & -1 \\
\hline Middle Occipital Gyrus (MOG) & 37 & $\mathbf{R}$ & 44 & -64 & -3 & 37 & $\mathbf{R}$ & 44 & -64 & -3 & 37 & $\mathbf{R}$ & 44 & -64 & -3 \\
\hline & & $\mathbf{L}$ & -44 & -69 & 0 & & $\mathbf{L}$ & -44 & -69 & 0 & & $\mathbf{L}$ & -44 & -69 & 0 \\
\hline
\end{tabular}

\section{FDR $\mathrm{q}=\mathbf{0 . 0 0 5}$}

FDR $q=0.01$

FDR $q=0.05$

In the frontal lobe, the activation of area 6 in the left medial frontal gyrus (MFG; Figures 2, A and D, yellow arrows) in c1 and c6 was observed; in $\mathrm{cl}$ and c6 activated foci were present in the precentral gyrus (PrG; lateral area 6) in both hemispheres, in the right area 9 of the inferior frontal gyrus (IFG; Tables 2 and 3) and in left area 45 of the inferior frontal gyrus (triangularis pars, TrIFG), exclusively in c6.

In the parietal lobe, activation was observed in precuneus $(\mathrm{PrCu}$; $\mathrm{BA7}$ ) in the right hemisphere in $\mathrm{c} 1$ and $\mathrm{c} 3$; in the superior parietal lobule (SPL; area 7) the activation was bilateral in $\mathrm{cl}$ and in the left side in c6 (Tables 2 and 3). In c1 and c6 the activation of left area 40 of the inferior parietal lobule (supramarginal gyrus, SMG,) was observed.

In all conditions the activation of area 22 (temporo-parietal junction, TPJ) in the right superior temporal gyrus (STG) was reported
(Tables 2 and 3), and in the left hemisphere too in c2 and c3 (Table 3). Bilateral activation was observed in area 39 (angular gyrus, AngG) of the superior temporal gyrus in $\mathrm{cl}$ (Table 2), and in the right hemisphere in c2, c3 (Table 3), c5, c6 and c7.

In the insular lobe, the activation of posterior area 13 was observed in both hemispheres in c1-c3 (Tables 2 and 3), and c5-c7, in the left hemisphere only in $\mathrm{c} 4$.

In all conditions, activation of visual areas of the occipital lobe were observed in both hemispheres (summarized in Table 2; Figure 2, D and $\mathrm{G})$ : cuneus (Cun; area 17 and area 18), lingual gyrus (LinG; area 18), middle occipital gyrus (MOG; area 19 and area 37). No BOLD signal in the corpus callosum was observed (Figure 3A). 


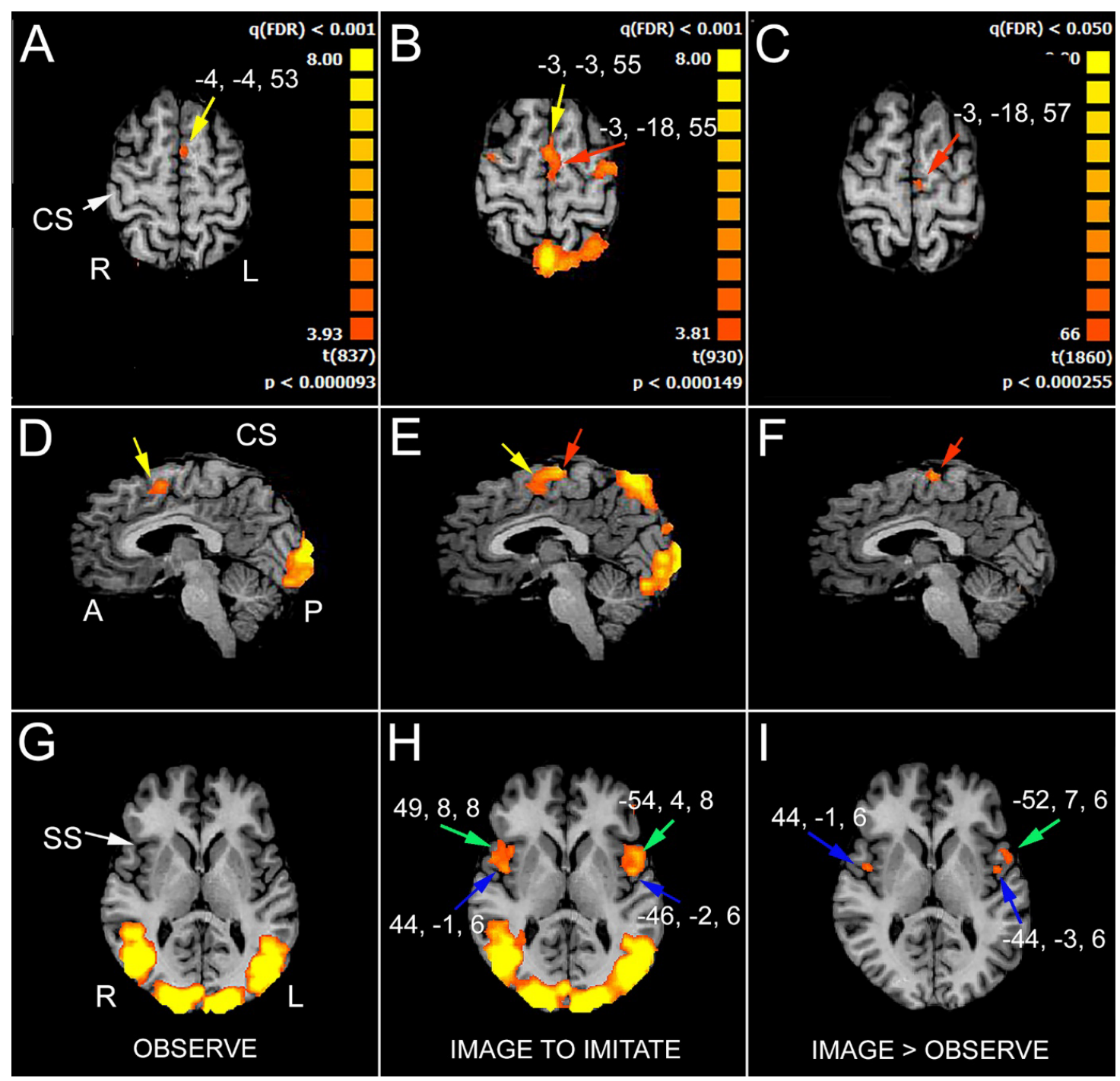

Figure 2. Significant activation in the different runs. A, D, G, OBS condition: the activation of the anterior part of the left supplementary motor area (SMA, yellow arrows in A and D) is evident, as well as the activation of visual areas in the occipital cortex (D and G). B, E, H, IIMsL condition: the activation of the anterior (yellow arrows) and posterior left SMA (red arrows) is evident in B and E, as well as the activation of visual areas in the occipital cortex (E and H). In $\mathrm{H}$ are also visible the activation foci in IFG (area 44, green arrows) and opercular cortex (blue arrows) in both hemispheres. C, F, I, IIMsL $>$ OBS condition: only the activation foci in the left posterior SMA (C and F, red arrows), left IFG (I, green arrow) and bilateral parietal opercula (I, blue arrows) are evident. In each condition, axial images are from two different $\mathrm{z}$ values: in $\mathrm{A}$ and $\mathrm{G}$, from OBS condition, $\mathrm{z}=53$ and 6 , respectivly; in B and $\mathrm{H}$, from IIMsL condition, $\mathrm{z}=55$ and 6 , respectively; in $\mathrm{C}$ and $\mathrm{I}$, from IIMsL $>\mathrm{OBS}$ condition, $\mathrm{z}=57$ and 6 , respectively. In $\mathrm{D} \mathrm{x}=-4$, in $\mathrm{E}$ and $\mathrm{F} \mathrm{x=-3}$. CS, central sulcus; SS, sylvian sulcus; according to the radiological convention, the left hemisphere is shown on the right

\section{Brain areas activted during IIMsL task}

During IIMsL run, the contrast task IIMsL versus Baseline resulted in map of active voxel clusters, whose Talairach coordinates are reported in Tables 2 and 4 . Multisubject analysis generated 41593 active voxels, to which the FDR statistical approach was applied, with $\mathrm{q}=0.001$ and cluster threshold $=10$ voxels. Additional analyses were performed with less selective, but still significant, values of $\mathrm{q}$, which was $0.005,0.01$ and 0.05 . In Table 4 , the results obtained with less selective $q$ values are evidenced.

In the frontal lobe activation foci were observed in MFG (area 6; Figure 2, B and E, yellow and red arrows), bilaterally in all conditions (Table 4). Also activation in PrG (lateral area 6) was evident in both hemispheres in all conditions, although at different $\mathrm{q}$ values (Figure 2B; Table 4); activation was also observed in IFG in area 9, bilaterally in $c 1-c 3$ and $c 6$ and $c 7$, in the left hemisphere in $c 4$ and c5. Bilateral activation was also observed area 44 of the inferior frontal gyrus (opercularis pars, OpIFG; Figure 2H, green arrow) in all conditions, although at different $\mathrm{q}$ values (Table 4); in the right pars triangularis of the inferior frontal gyrus (area 45) activation was observed in all conditions, although at different $\mathrm{q}$ values (Table 4). In area 46 of the prefrontal gyrus (dorsolateral prefrontal cortex; DLPFC) a left hemisphere activation was observed in all conditions except $c 7$.

In the parietal lobe, consistent activation was observed in all conditions in the left PrCun (area 7; Figure 2B); bilateral activation was observed in the superior parietal lobule (SPL; area 7) in c1, c2 and $c 6$, and in the left hemisphere in c3-5 and 7; bilateral activation was also observed in the inferior parietal lobule (IPL; area 40) in c1, c2 (Tables 2 and 4), c6 and c7, and in the left hemisphere in c3, c4 and c5 (Table 4).

In the temporal lobe the activation of area 22 in the STG (TPJ) was bilateral in $\mathrm{c1}$ and $\mathrm{c} 2$, and right in $\mathrm{c} 3-\mathrm{c} 7$ (Tables 2 and 4); a bilateral activation was also observed in area 39 (AngG) in all conditions. 
In the insular lobe, the activation of posterior area 13 was in both hemispheres in all conditions (Table 4). The most interesting observation was however the activation of the middle insula (area 13) in both hemispheres in all conditions (Figure $2 \mathrm{H}$, blue arrows; Table 4).

In the occipital lobe, the activation of visual areas was observed in both hemispheres in all conditions (Table 2; Figure 2, E and H): areas $17,18,19$ and 37.

Activation was also observed within the corpus callosum (Figure $3 \mathrm{~B}$ ), in the anterior and middle portion of the body, at a $\mathrm{p}$ value (non FDR) $\mathrm{B}<0.035$.

Table 4. Cortical areas activated in IIMsL

\begin{tabular}{|c|c|c|c|c|c|c|c|c|c|c|c|c|c|c|c|}
\hline \multirow{3}{*}{\begin{tabular}{|c|} 
IMAGE TO IMITATE, FDR $\mathbf{q}=\mathbf{0 . 0 0 1}$ \\
\\
Cortical Area \\
\end{tabular}} & \multirow[b]{3}{*}{ BA } & \multicolumn{4}{|c|}{$\begin{array}{l}\text { All gestures } \\
\text { condition } 1\end{array}$} & \multicolumn{5}{|c|}{ Model's RL gestures condition 2} & \multicolumn{5}{|c|}{ Model's LL gestures condition 3} \\
\hline & & \multirow[b]{2}{*}{$\mathbf{H}$} & \multicolumn{3}{|c|}{ Talairach coordinates } & \multirow[b]{2}{*}{$\mathbf{B A}$} & \multirow[b]{2}{*}{$\mathbf{H}$} & \multicolumn{3}{|c|}{ Talairach coordinates } & \multirow[b]{2}{*}{ BA } & \multirow[b]{2}{*}{$\mathbf{H}$} & \multicolumn{3}{|c|}{ Talairach coordinates } \\
\hline & & & $\mathrm{x}$ & $\mathrm{y}$ & $\mathrm{z}$ & & & $\mathrm{x}$ & $\mathrm{y}$ & $\mathrm{z}$ & & & $\mathrm{x}$ & $\mathrm{y}$ & $\mathrm{z}$ \\
\hline \multicolumn{16}{|l|}{ FRONTAL LOBE } \\
\hline \multirow[t]{2}{*}{ Medial Frontal Gyrus (MFG) } & 6 & $\mathbf{R}$ & 3 & -5 & 58 & 6 & $\mathbf{R}$ & 3 & -5 & 58 & 6 & $\mathbf{R}$ & 3 & -5 & 58 \\
\hline & & $\mathbf{L}$ & -2 & -9 & 58 & & $\mathbf{L}$ & -2 & -9 & 58 & & $\mathbf{L}$ & -2 & -9 & 58 \\
\hline \multirow[t]{2}{*}{ Inferior Frontal Gyrus (IFG) } & 9 & $\mathbf{R}$ & 29 & 31 & 27 & 9 & $\mathbf{R}$ & 29 & 31 & 27 & 9 & $\mathbf{R}$ & 29 & 31 & 27 \\
\hline & & $\mathbf{L}$ & -39 & 30 & 27 & & $\mathbf{L}$ & -39 & 29 & 27 & & $\mathbf{L}$ & -39 & 30 & 27 \\
\hline \multirow[t]{2}{*}{ Inferior Frontal Gyrus (IFG) } & 44 & $\mathbf{R}$ & 49 & 8 & 8 & 44 & $\mathbf{R}$ & 49 & 8 & 8 & 44 & $\mathbf{R}$ & 49 & 13 & 4 \\
\hline & & $\mathbf{L}$ & -54 & 4 & 8 & & $\mathbf{L}$ & -41 & 12 & 8 & & $\mathbf{L}$ & -48 & 9 & 4 \\
\hline \multirow[t]{2}{*}{ Inferior Frontal Gyrus (IFG) } & 45 & $\mathbf{R}$ & 44 & 14 & -1 & 45 & $\mathbf{R}$ & 44 & 14 & -1 & 45 & $\mathbf{R}$ & 44 & 14 & -1 \\
\hline & & $\mathbf{L}$ & & & & & $\mathbf{L}$ & & & & & $\mathbf{L}$ & & & \\
\hline Inferior Frontal Gyrus (IFG) & 46 & $\mathbf{R}$ & & & & 46 & $\mathbf{R}$ & & & & 46 & $\mathbf{R}$ & & & \\
\hline & & $\mathbf{L}$ & -43 & 43 & 9 & & $\mathbf{L}$ & -41 & 41 & 9 & & $\mathbf{L}$ & -44 & 41 & 8 \\
\hline Precentral Gyrus (PrG) & 6 & $\mathbf{R}$ & 43 & -2 & 43 & 6 & $\mathbf{R}$ & 44 & -6 & 43 & 6 & $\mathbf{R}$ & 43 & -2 & 43 \\
\hline & & $\mathbf{L}$ & -42 & -6 & 43 & & $\mathbf{L}$ & -44 & -6 & 43 & & $\mathbf{L}$ & -42 & -6 & 43 \\
\hline PARIETAL LOBE & & & & & & & & & & & & & & & \\
\hline Parietal lobe, precuneus & 7 & $\mathbf{R}$ & & & & 7 & $\mathbf{R}$ & & & & 7 & $\mathbf{R}$ & & & \\
\hline & & $\mathbf{L}$ & -2 & -70 & 51 & & $\mathbf{L}$ & -2 & -70 & 51 & & $\mathbf{L}$ & -2 & -70 & 51 \\
\hline Superior parietal lobule (SPL) & 7 & $\mathbf{R}$ & 32 & -65 & 47 & 7 & $\mathbf{R}$ & 32 & -61 & 47 & 7 & $\mathbf{R}$ & & & \\
\hline & & $\mathbf{L}$ & -30 & -70 & 47 & & $\mathbf{L}$ & -30 & -70 & 47 & & $\mathbf{L}$ & -30 & -70 & 47 \\
\hline Inferior Parietal Lobule (IPL) & 40 & $\mathbf{R}$ & 41 & -46 & 47 & 40 & $\mathbf{R}$ & 39 & -47 & 48 & 40 & $\mathbf{R}$ & & & \\
\hline & & $\mathbf{L}$ & -39 & -56 & 47 & & $\mathbf{L}$ & -40 & -54 & 48 & & $\mathbf{L}$ & -54 & -44 & 27 \\
\hline TEMPORAL LOBE & & & & & & & & & & & & & & & \\
\hline Superior temporal gyrus (STG) & 22 & $\mathbf{R}$ & 45 & -44 & 12 & 22 & $\mathbf{R}$ & 45 & -44 & 12 & 22 & $\mathbf{R}$ & 47 & -44 & 11 \\
\hline & & $\mathbf{L}$ & -56 & -42 & 12 & & $\mathbf{L}$ & -56 & -42 & 12 & & $\mathbf{L}$ & & & \\
\hline Temp Lobe, sup temp gyrus & 39 & $\mathbf{R}$ & 40 & -51 & 7 & 39 & $\mathbf{R}$ & 40 & -55 & 5 & 39 & $\mathbf{R}$ & 42 & -58 & 7 \\
\hline & & $\mathbf{L}$ & -50 & -54 & 7 & & $\mathbf{L}$ & -50 & -54 & 7 & & $\mathbf{L}$ & -50 & -54 & 7 \\
\hline OCCPITAL LOBE & & & & & & & & & & & & & & & \\
\hline Cuneus (Cun) & 17 & $\mathbf{R}$ & 12 & -94 & 1 & 17 & $\mathbf{R}$ & 12 & -94 & 1 & 17 & $\mathbf{R}$ & 12 & -94 & 1 \\
\hline & & $\mathbf{L}$ & -12 & -94 & 1 & & $\mathbf{L}$ & -12 & -94 & 1 & & $\mathbf{L}$ & -12 & -94 & 1 \\
\hline Cuneus (Cun) & 18 & $\mathbf{R}$ & 10 & -91 & 18 & 18 & $\mathbf{R}$ & 10 & -91 & 18 & 18 & $\mathbf{R}$ & 10 & -91 & 18 \\
\hline & & $\mathbf{L}$ & -9 & -92 & 18 & & $\mathbf{L}$ & -9 & -92 & 18 & & $\mathbf{L}$ & -9 & -92 & 18 \\
\hline Lingual Gyrus (LgG) & 18 & $\mathbf{R}$ & 20 & -77 & -9 & 18 & $\mathbf{R}$ & 1 & -83 & 3 & 18 & $\mathbf{R}$ & 20 & -77 & -9 \\
\hline & & $\mathbf{L}$ & -20 & -77 & -9 & & $\mathbf{L}$ & -2 & -83 & 3 & & $\mathbf{L}$ & -20 & -77 & -9 \\
\hline Middle Occipital Gyrus (MOG) & 19 & $\mathbf{R}$ & 47 & -61 & 6 & 19 & $\mathbf{R}$ & 47 & -61 & 6 & 19 & $\mathbf{R}$ & 47 & -61 & 6 \\
\hline & & $\mathbf{L}$ & -46 & -69 & 6 & & $\mathbf{L}$ & -46 & -69 & 6 & & $\mathbf{L}$ & -46 & -69 & 6 \\
\hline Middle Occipital Gyrus (MOG) & 37 & $\mathbf{R}$ & 44 & -64 & -3 & 37 & $\mathbf{R}$ & 44 & -64 & -3 & 37 & $\mathbf{R}$ & 44 & -64 & -3 \\
\hline & & $\mathbf{L}$ & -44 & -69 & 0 & & $\mathbf{L}$ & -44 & -69 & 0 & & $\mathbf{L}$ & -44 & -69 & 0 \\
\hline INSULAR LOBE & & & & & & & & & & & & & & & \\
\hline Insula (Ins) middle & 13 & $\mathbf{R}$ & 44 & -1 & 6 & 13 & $\mathbf{R}$ & 44 & 1 & 6 & 13 & $\mathbf{R}$ & 44 & 1 & 6 \\
\hline & & $\mathbf{L}$ & -46 & -2 & 6 & & $\mathbf{L}$ & -46 & 5 & 6 & & $\mathbf{L}$ & -46 & -3 & 6 \\
\hline Insula (Ins) posterior & 13 & $\mathbf{R}$ & 44 & -39 & 21 & 13 & $\mathbf{R}$ & 47 & -40 & 20 & 13 & $\mathbf{R}$ & 51 & -40 & 20 \\
\hline & & $\mathbf{L}$ & -49 & -44 & 22 & & $\mathbf{L}$ & -51 & -44 & 20 & & $\mathbf{L}$ & -51 & -46 & 20 \\
\hline Corpus Callosum (CC) non fdr, $p<0.045$ & & & -2 & 0 & 23 & & & 10 & -29 & 22 & & & -1 & 1 & 21 \\
\hline FDR $q=0.005$ & & & & & & & & & & & & & & & \\
\hline FDR $q=0.05$ & & & & & & & & & & & & & & & \\
\hline
\end{tabular}

\section{Brain areas activated resulting from the difference between IIMsL versus OBS task}

A further analysis was performed on the pattern of activation obtained by the contrast of IIMsL versus the OBS tasks. A map of active voxel clusters resulted, whose Talairach coordinates are reported in Table 2. Multisubject analysis generated 41052 active voxels, to which the FDR statistical approach was applied, with $\mathrm{q}=0.05$ and cluster threshold $=3$ voxels. In some cases, additional analyses were performed without FDR statistical approach, to evidence also smaller activations; in that case, the $\mathrm{p}$ values were $\leq 0.01$ or $\leq 0.05$. 


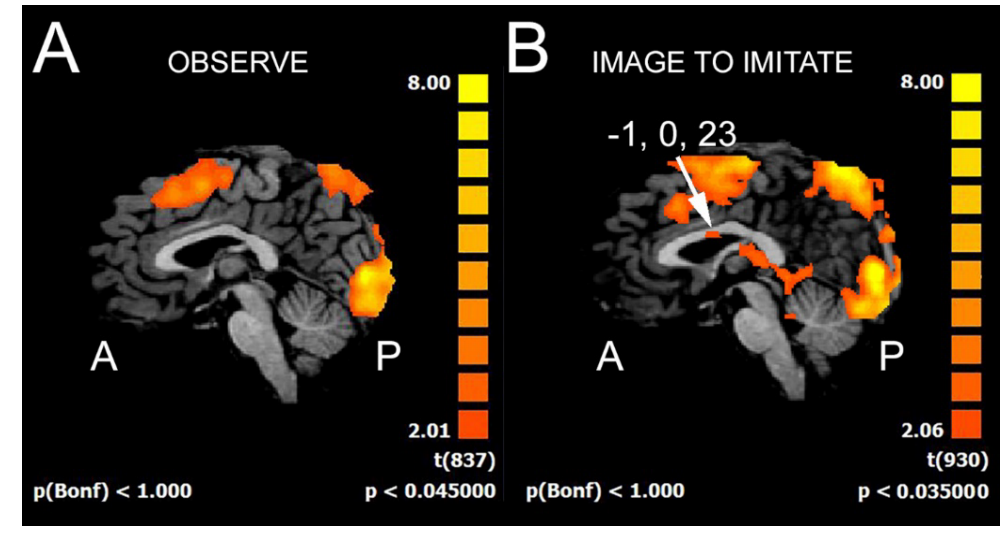

Figure 3. Activation in the corpus callosum in the different runs. A, OBS condition: no activation is evident in the CC. B, IIMsL condition: an activation focus is visible in the anterior portion of the callosal body. The numbers above the arrows in frame B indicate the Talairach coordinates of the activation foci. A, anterior; $\mathrm{P}$, posterior

In the frontal lobe, in all conditions, activation of MFG (area 6) in the left hemisphere was reported, although with different $\mathrm{p}$ values (Figure 2, C and F, red arrows; Table 2). In c4 only the activation of left anterior prefontal cortex areas 8 (superior portion) and 10 (inferior portion) was also observed. The activation was present in area 44 of the left inferior frontal gyrus (OpIFG) in all conditions, although with different $\mathrm{p}$ values, and in the right side in $\mathrm{c} 5$ and c6 (Figure 2I, green arrow; Table 2).

In the parietal lobe, activation was present in area 7 of left SPL in c1 and 4 , in the left precuneus ( $\mathrm{PrCu}$ ) in $\mathrm{c} 4$, and in the left area 40 of the IPL in c1 (Table 2) and c6.

Activation of area 22 in the STG was in the right hemisphere in c1-c3 (Table 2); bilateral activation was found in area 41 of the inferior temporal gyrus (ITG) in $\mathrm{c} 2$, and in the left hemisphere only in $\mathrm{c}$. Bilateral activation of the middle insula (area 13) was observed in all conditions, although with different $\mathrm{p}$ values, except in $\mathrm{c} 7$, where the activation was only in the left hemisphere (Figure 2I, blue arrows; Table 2). Activation was also observed within the corpus callosum, in the middle portion of the body, at a p value (non FDR) $<0.045$.

\section{Discussion}

The present study was aimed to identify, in healthy control subjects: 1. the cortical areas activated during the observation of intransitive meaningful gestures performed by a model, presented in video clips in $3^{\text {rd }}$ person perspective; 2 . the cortical areas activated by imaging to imitate the same gestures using the same limb of the model. To compare present functional data with previous behavioural data, present study was carreid out in the the same group of subjects participating in previous behavioural study [3].

Previous behavioral studies investigated the strategies used by healthy subjects (controls; [3] and callosotomized patients in imitating intransitive gestures [8]. Results of these studies demonstrated that: 1 . in free sessions the mirror mode of imitation was preferred from both control subjects $(61 \%)$ and patients $(66 \%) ; 2$. in driven sessions the anatomical mode is privileged by controls (93\%), but not by patients, who still preferred mirror mode (61\%); 3 . in driven sessions, the terms "same" and "opposite" were interpreted according to an anatomical criterion by control subjects; likely, according to a spatial criterion, in patients. In addition, hand preference did not correlate with action observation and execution (imitative act; see $[3,8]$.

As explained in the Introduction, being the present work performed within the magnet, the stimlation protocol required some adaptaions, since during a fMRI run it is necessary to avoid any movement. Therefore, the OBS and IIMsL modality have been used, and it was assumed that the OBS modality could be similar to the free imitation (occurring in our previous study in a mirror mode, also in healthy subjects), and that driven imitation could be substitute in the magnet by an imagined imitation with the same limb as the model, in that from our previous paper it was evident that healthy subjects performed driven imitation in anatomical mode [3].

\section{Stimuli and instruction variables}

The stimuli used in the present functional study were two meaningful gestures, one body-related (the silence gesture) and one body-unrelated (the bye-bye gesture), performed by the model with her right or left upper limb, as in the previous studies $[3,8]$. These two meaningful gestures have been selected since no differences in performance were previously observed between meaningful and meaningless gestures; in addition, the silence and bye-bye gestures are well kown acts and consequently easier to be executed, even with imagination.

Meaningful gestures are familiar communicative acts, that usually accompany specific words, they are universally shared, and their execution is frequently associated with speech production [23]. Their motor program is stored in memory and it can be retrieved in everyday life to execute the movement; however, in order to meet the specific demand (i.e. to imitate a gesture using the same limb of a model), movement parameters have to be specified. This is the case of IIMsL task, where subjects need to mentally evaluate how to move limbs respect to one's own body space and to the outside space before performing. Differently from OBS task, where the request is simply to observe gestures shown in the video clips, in IIMsL the demande becomes enigmatic as the participat has first 1. to interpret the meaning of the concept "same", and afterwards 2. to recruit the exact limb and sequence of movements between motor programs. According to the aim of the present study, the step relative to the attribution of a meaning to the term "same" is crucial as strictly linked to the anatomical imitation aspect, so that it appears inseparable from the concepts of "body schema", referring to the interaction between the subject and the environment and providing body with external spatial coordinates, and from the concepts of "body image", relating to body ownership and selfconsciousness.

\section{Observe run (OBS)}

In this condition, consistent activation was observed in the visual areas of occipital lobes of both hemispheres, as expected, since the 
visual stimuli were presented centrally in the visual field. In addition, during the OBS run, the recruitmet of the cortical regions belonging to the MNS occurs: the left medial area 6 in the MFG, bilateral precentral motor cortex (PMC, area 4), left inferior parietal lobule (SMG, area 40), and bilateral angular gyrus (AngG, areas 39); all these areas built a fronto-parietal network known as the observation-execution matching system having the role to recognize the action [24,25] and to be the neural substrate that underlies understanding and eventually imitating actions. The other activated areas, i.e., left IPL (area 40) and right IFG (area 9), are likely related to the hand and finger posture, respectively [26]; the bilateral activation of the posterior part of SPL (area 7), seems to be specifically involved in the control of visual guided movements (see data and literature in [27]); finally the activation of the right TPJ (posterior area 22), being likely related to the recognition of self/others body parts and movements, could have the funcion to recognize which body region of the model has been used to produce the gesture [28].

Since the fMRI temporal resolution is low, it was not possible to define the order of activation, if any. The present findings are in agreement with previous observations, reporting that the network of motor areas involved in preparation and execution of action was also activated by simple observation of actions [12]. In addition, observing an action performed by another person activates neural circuits that are very similar to those used in performing the same action and facilitates the execution of the observed movements (see data and literature in [29].

\section{Image to imitate with the same limb run (IIMsL)}

In this conditions, other than the above mentioned activation pattern, a more extensive activation was observed in the right TPJ incuding larger portion of area 39 (=angular gyrus, involved in the rightleft recognition; [30]; the activation in the MFG and IFG was bilateral. Moreover, activation appeared also in the left hemisphere in the pars triangularis of the inferior frontal gyrus (area 45; recognition of the meaning), bilaterally in the opercular part of the inferior frontal gyrus (area 44) and in the insula in the parietal opercula. A similar pattern of activation was previously described during imagery imitation of hand position [31]; the more extenisve activation evoked by motor imagery rather than simple action observation was also previously reported in healthy subjects for object-related hand actions [13].

To image imitating a gesture with the same limb of a model, a subject first of all has to observe the gesture. This step would activate the mirror neuron system, likely to recognize the action and the body region used to perform the gesture [32]. Such an activation could be the same as observed during the OBS task. In addition, to use the same limb, the subject will have first to recognize the limb which has been used by the model, i.e., if her arm or leg, and of which side, if her right or left. To do this, likely the TPJ and IPL will be activated, dealing with the recognition of the body part and with the shift from self to other body part [28]. Later, the subject will have to decide what the term "same" means: adult control subjects interpreted this instruction according to an anatomical criterion, as also demonstrated previously in a behavioural context [3]. Finally, they will expect to activate the area(s) providing the motor program for the imaged movement according to the selected anatomical criterion: this area(s) could be the parietal operculum(a), likey involved into the activation of selected limb (see below).

\section{IIMsL task versus OBS task}

This condition, evidencing cortical areas mostly activted during IIMsL task versus OBS, confirms and reinforces the differences described above: activation was observed in left area 6 of MFG (posterior SMA or SMA proper), left area 40 of IPL, and left area 44 of IFG, in the right TPJ (posterior area 22), and bilaterally in the insula, in the parietal opercula (PO).

Subjects of the present study referred they imagined to imitate with an anatomical perspective, therefore the activation of the above mentioned areas could be considered specifically related to the anatomical imitation.

\section{Neural mechanism: comparison with other studies}

By analyzing the results of the present research also in the light of previous functional study carried out in similar condition, it can be hypothesized that, during the anatomical imitation of intransitive upper limb gesures, the left area 6, left area 40 of IPL, and left area 44 of IFG, right posterior area 22 of TPJ, and PO bilaterally in the insula are specifically activated, likely assuming the following roles:

1. the posterior SMA (area 6) would represent the spatial trajectory of the gesture to be imitated, also independently from the body part executing that gesture, as recently shown [33]. This recent finding is in agreement with previous report from brain damaged patients, who showed deficits in anatomical imitation specially when lesion involved the left dorsal premotor cortex [34]; 2. the left area 44 would recognize and understand the observed action and transfer the information to the TPJ [35]. The activation of area 44 has been observed in previous hand [36] and finger [35] posture imitation studies; 3. the TPJ would allow to shift from other/self gesture recognition [37]; TPJ has been often observed to be involved in gesture imitative behaviour (see [38] for a review), as well as IPL for hand gesture imitation [26], mainly of meaningful gestures [39], in particular in the left hemisphere [40]. The activation of TPJ has been recently described during imitative tasks, and has been defined as involved in the control of the imitation in case of spatial incompatibility [41]; 4. the bilateral activation of the parietal opercula during the anatomical imitation of finger position was recently reported in healthy subjects [42], leading the Authors hyopthesize that the PO is "implicated (together with parietal, prefrontal and insular structures) in imitation tasks and, in particular, in coding the body part that executes an action, and not the action itself" [42] (see below). Other studies point to a role for the PO in the anatomical imitation, either in the right hemisphere [43] or in both [44]. In particular, a TMS interference with the parietal opercula's activity modulated the imitative compatibility but not the spatial compatibility, suggesting that these two processes are likely to be independent [44]. Although these studies describe finger position and not gesture imitation, it is reasonable to believe that the neural mechanism involved is likely the same.

It has been recently shown that a subportion of the parietal operculum, named OP 4, is strongly connected with premotor and primary motor cortex, suggesting an interaction with the motor system [45]. Therefore, the parietal operculum can be considered as a crucial interface point, in which, during the imitation behaviour, the information about the body part in the space of the model is compared with the orientation in the space of the body of the performer. It is worth noticing that this region has also been associated with the processing of the body schema, thus suggesting a connection of the imitation network with the body representations [46].

A very recent review analyzing the neural correlates of the imitation of intransitive gestures [7] confirmed that "imitation is a complex function sustained by a network of bilateral brain areas", in which the 
left hemisphere, more particularly the parietal cortex, has a key role in imitation of intransitive gestures. However, further studies are required to disentangle the role of right brain structures in imitation, in the parietal (SMG, AG and SPL) and in the frontal lobes (IFG and insula).

\section{Conclusion}

In conclusion, present findings confirm and extend current understanding of the neural mechanism of anatomical imitation, and provide additional supports about the role of parietal opercula. Indeed, our results indicate that the imitation according to an anatomical criterion seems to require the cooperation of cortical areas in both hemispheres: the MGF, IPL and IFL in the left, the TPJ in the right, and the PO in both hemispheres. This final observation is in agreement with previous studies (reviewed in [6,7]; it is also consistent with our previous behavioural results obtained in callosotomized patients [8], according to which these patients performed the anatomical imitation less frequently than control subjects, in that they have more difficulty to engage both hemispheres in a coordinated activity. It seems reasoneable to hypothesize, therefore, that the different performances of the patients could be ascribed to the interruption of the callosal fibres and consequently to the impossibility for the hemispheres to communicate and cooperate.

Dorsal premotor area 6 communicate with the other hemisphere by sending fibers in the central portion of the CC [47]. Area 44 of IFG sends interhemispheric fibres through the ventral rostral body and ventral anterior midbody [47]. Callosal fibres arising from IPL (areas 39-40) cross the dorsal splenium, and those from TPJ (posterior area 22 and 39) cross the ventral splenium [47]. No conclusive data are available about the trajectory of interhemispheric fibres connecting the opercular cortices of the two sides, although data from studies on gustatory (frontoparietal operculum; [48]) and tactile (parietal operculum; [49-54] interhemispheric transfer seem to indicate the anterior and central callosal body, respectively. Accordingly, patients lacking callosal fibres from anterior and/or central body and/or splenium display a lower proportion of anatomical imitation performance [8]. Further studies will be necessary to clarify this issue.

\section{Aknowledgements}

The authors are especially grateful to all subjects for participating in the study, and to the radiology technicians Luigi Imperiale and Lucio Montesi for their invaluable help in collecting data during and soon after the volunteers' fMRI sessions.

\section{Funding}

This work was supported by Ministero Istruzione, Università e Ricerca, PRIN 2009, and by Università Politecnica delle Marche, RSA 2010, RSA 2011.

\section{Declaration of interest}

We wish to confirm that there are no known conflicts of interest associated with this publication and there has been no significant financial support for this work that could have influenced its outcome.

\section{References}

1. Farmer H, Ciaunica A, Hamilton AFdeC (2018) The functions of the imitative behaviour in humans. Mind Lang 33: 378-396.

2. Chaminade T, Meltzoff AN, Decety J (2005) An fMRI study of imitation: action representation and body schema. Neuropsychologia 43: 115-127.
3. Pierpaoli C, Ferrante L, Manzoni T, Fabri M (2014) Anatomical or mirror - mode imitation? A behavioral approach. Arch Ital Biol 152: 20-31.

4. Meltzoff NA, Moore MK (1977) Imitation of Facial and Manual Gestures by Human Neonates. Science 198: 75-78.

5. Wapner S, Cirillo L (1968) Imitation of a model's hand movements: age changes in transposition of left-right relations. Child Dev 39: 887-894.

6. Caspers S, Zilles K, Laird AR, Eickhoff SB (2010) ALE meta-analysis of action observation and imitation in the human brain. NeuroImage 50: 1148-1167.

7. Lesourd M, Osiurak F, Baumard J, Bartolo A, Vanbellingen T, et al. (2018) Cerebral correlates of imitation of intransitive grestures: an integrative review of neuroimaging data and brain lesion studies. Neurosci Biobehav Rev 95: 44-60.

8. Pierpaoli C, Foschi N, Cagnetti C, Ferrante L, Manzoni T, et al. (2018) Imitation strategies in callosotomized patients. Arch Ital Biol 156: 12-26.

9. Iacoboni M, Woods RP, Brass M, Bekkering H, Mazziotta JC, et al. (1999) Cortical mechanisms ofh uman imitation. Science 286: 2526-2528.

10. Rizzolatti G, Fogassi L, Gallese V (2001) Neurophysiological mechanisms underlying the understanding and imitation of action. Nat Rev Neurosci 2: 661-670.

11. Rizzolatti G, Craighero L (2004) The Mirror-Neuron System. Annu Rev Neurosci 27 169-192.

12. Calvo-Merino B, Glaser DE, Grèzes J, Passingham RE, Haggard P, et al. (2005) Action observation and acquired motor skills: an fMRI study with expert dancers. Cer Cor 15: $1243-1249$.

13. Nedelko V, Hassa T, Hamzei F, Schoenfeld MA, Dettmers C, et al. (2012) Action imagery combined with action observation activates more corticomotor regions than action observation alone. J Neurol Phys Ther 36: 182-188.

14. David N, Bewernick BH, Cohen MX, Newen A, Lux S, et al. (2006) Neural representations of Self versus Other: visual-spatial perspective taking and agency in a virtual ball-tossing game. J Cogn Neurosci 18: 898-910.

15. David N, Aumann C, Santos NS, Bewernick BH, Eckhoff SB, et al. (2008) Differentia involvement of the posterior temporal cortex in mentalizing but not perspective taking. Soc Cogn Affect Neurosci 3: 279-289.

16. Milivojevic B, Hamm JP, Corballis MC (2008) Functional neuroanatomy of mental rotation. J Cogn Neurosci 21: 945-959.

17. Zacks JM (2008) Neuroimaging studies of mental rotation: a meta-analysis and review. J Cogn Neurosci 20: 1-19.

18. Pierpaoli C, Berlucchi G, Manzoni T, Fabri M (2009) A behavioral study of imitation of intransitive meaningful and meaningless gestures. XIII National Congress of the Italian Society for Neuroscience, Milano, 2-5 October.

19. Pierpaoli C, Berlucchi G, Paggi A, Ferrante L, Manzoni T, et al. (2010a) Aatomical and mirror imitation strategy in callosotomized patients. $61^{\text {st }}$ National Congress of Società Italiana di Fisiologia, Varese, September 15-17.

20. Pierpaoli C, Berlucchi G, Paggi A, Manzoni T, Fabri M, et al. (2010b) A behavioral study of gesture imitation: an anatomical or mirror-image strategy? $7^{\text {th }}$ FENS Forum of European Neuroscience Societies, Amsterdam, July 3-7.

21. Oldfield RC (1971) The assessment and analysis of handedness: the Edinburgh Inventory. Neuropsychologia 9: 97-113.

22. Talairach J, Turnoux P (2008) Co-Planar Stereotaxic Atlas of the Human Brain. New York (USA): Thieme Medical Publishers.

23. Goldin-Meadow S (1999) The role of gesture in communication and thinking. Trends Cogn Sci 3: 419-429.

24. Rizzolatti G, Cattaneo L, Fabbri-Destro M, Rozzi S (2014) Cortical mechanisms underlying the organization of goal-directed actions and mirror neuron-based action understanding. Physiol Rev 94: 655-706.

25. Rizzolatti G, Sinigaglia C (2016) The mirror mechanism: a basic principle of brain function. Nat Rev Neurosci 17: 757-765.

26. Goldenberg G, Karnath H-O (2006) The neural basis of imitation is body part specific. J Neurosci 26: 6282-6287.

27. Wang J, Yang Y, Fan L, Xu J, Changhai L, et al. (2015) Convergent functional architecture of the superior parietal lobule unraveled with multimodal neuroimaging approaches. Hum Brain Mapp 36: 238-257. 
28. Overney LS, Michel CM, Harris IM, Pegna AJ (2005) Cerebral processes in mental transformations of body parts: recognition prior to rotation. Brain Res Cogn Brain Res 25: $722-734$.

29. Vingerhoets G, Stevens L, Meesdom M, Honoré P, Vandemaele P, et al. (2012) Influence of perspective on the neural correlates of motor resonance during natural action observation. Neuropsychol Rehabil Intern J 22: 752-767.

30. Seghier ML (2013) The angular gyrus: multiple functions and multiple dubdivisions. The Neuroscientist 19: 43-61.

31. Macuga KL, Frey SH (2012) Neural representations involved in observed, imagined, and imitated actions are dissociable and hierarchically organized. NeuroImage 59: 2798-2807.

32. Carmo JC, Rumiati RI, Vallesi A (2012) Understanding and imitating unfamiliar actions: distinct underlying mechanisms. PLoS ONE 7: e46939.

33. Wong AL, Jax SA, Smith LL, Buxbaum LJ, Krakauer JW, et al. (2019) Movement imitation via an abstract trajectory representation in dorsal premotor cortex. $J$ Neurosci 39: 3320-3331.

34. Chiavarino C, Apperly IA, Humphreys GW (2007) Exploring the functional and antomical bases of mirror-image and anatomical imitation: the role of the frontal lobes. Neuropsychologia 45: 784-795.

35. Molnar-Szakacs I, Iacoboni M, Koski L, Mazziotta JC (2005) Functional segregation within pars opercularis of the inferior frontal gyrus: evidence from fMRI studies of imitation and action observation. Cer Cor 15: 986-994.

36. Aziz-Zadeh L, Koski L, Zaidel E, Mazziotta J, Iacoboni M, et al. (2006) Lateralization of the human mirror neuron system. J Neurosci 26: 2964-2970.

37. Jackson PL, Meltzoff AN, Decety J (2006) Neural circuits involved in imitation and perspective-taking. NeuroImage 31: $429-439$.

38. Berlucchi G, Vallar G (2018) The history of the neurophysiology and neurology of the parietal lobe. In Handbook of Clinical Neurology, Elsevier 151: 3-30.

39. Lui F, Buccino G, Duzzi D, Benuzzi F, Crisi G, et al. (2008) Neural substrates for observing and imagining non-object-directed actions. Soc Neurosci 3 261-275.

40. Muhlau M, Hermsdörfer J, Goldenberg G, Wohlschläger AM, Castrop F, et al. (2005) Left inferior parietal dominance in gesture imitation: an fMRI study. Neuropsychologia 43: 1086-1098.

41. Swoden S, Catmur C (2015) The role of the right temporoparietal junction in the control of imitation. Cer Cor 25: 1107-1113.
42. Mengotti P, Corradi-Dell'Acqua C, Rumiati RI (2012) Imitation component in the human brain: an fMRI study. NeuroImage 59: 1622-1630.

43. Kubiak A, Kroliczak G (2016) Left extrastriate body area is sensitive to the meaning of symbolic gesture: evidence from fMRI repetition suppression. Sci Rep 6: 31064.

44. Mengotti P, Ticini LF, Waszak F, Schütz-Bosbach S, Rumiati RI, et al. (2013) Imitating others' actions: transcraniam magnetic stimulation on the parietal opercula reveals the processes underlying automatic imitation. Eur J Neurosci 37: 316-322.

45. Eickhoff SB, Jbabdi S, Caspers S, Laird AR, Fox PT, et al. (2010) Anatomical and functional connectivity of cytoarchitectonic areas within the human parietal operculum. J Neurosci 30: 6409-6421.

46. Corradi-Dell'Acqua C, Tomasino B, Fink GR (2009) What is the position of an arm relative to the body? Neural correlates of body schema and body structural description. JNeurosci 29: 4162- 4171 .

47. Chao Y-P, Cho K-H, Yeh C-H, Chou K-H, Chen J-H, et al. (2009) Probabilistic topography of human corpus callosum using cytoarchitectural parcellation and high angular resolution diffusion imaging tractography. Hum Brain Mapp 30: 3172-3187.

48. Mascioli G, Berlucchi G, Pierpaoli C, Salvolini U, Barbaresi P, Fabri M, Polonara G (2015) Functional MRI cortical activations from unilateral tactile-taste stimulations of the tongue. Physiol Behav 151: 221-229.

49. Fabri M, Polonara G, Quattrini A, Salvolini U, Del Pesce M, et al. (1999) Role of the corpus callosum in the somatosensory activation of the ipsilateral cerebral cortex: an fMRI study of callosotomized patients. Eur J Neurosci 11: 3983-3994.

50. Fabri M, Polonara G, Del Pesce M, Quattrini A, Salvolini U, et al. (2001) Posterior corpus callosum and interhemispheric transfer of somatosensory information: an fMRI and neuropsychological study of a partially callosotomized patient. $J$ Cogn Neurosci 13: 1071-1079.

51. Fabri M, Polonara G, Mascioli G, Paggi A, Salvolini U, et al. (2006) Contribution of the corpus callosum to bilateral representation of the trunk midline in the human brain a fMRI study of callosotomized patients. Eur J Neurosci 23: 3139-3148.

52. Fabri M, Polonara G, Mascioli G, Salvolini U, Manzoni T (2011) Topographica organization of human corpus callosum: an fMRI mapping study. Brain Res 1370: 99-111

53. Fabri M, Polonara G (2013) Functional topography of human corpus callosum: an FMRI mapping study. Neural Plast 251308

54. Polonara G, Mascioli G, Foschi N, Salvolini U, Pierpaoli C, et al. (2014) Further evidence for the topography and connectivity of the corpus callosum: an FMRI study of patients with partial callosal resection. $J$ Neuroimaging 25: 465-473.

Copyright: (C2020 Pierpaoli C. This is an open-access article distributed under the terms of the Creative Commons Attribution License, which permits unrestricted use, distribution, and reproduction in any medium, provided the original author and source are credited. 